\title{
Binder Jet 3D Printing of 316L Stainless Steel: Effects of HIP on Fatigue
}

Katerina Kimes $^{1 *}$, Kyle Myers ${ }^{2}$, Andrew Klein ${ }^{2}$, Magnus Ahlfors ${ }^{3}$, Erica Stevens ${ }^{1}$ and Markus Chmielus $^{1 *}$

1. Dept. of Mechanical Engineering and Materials Science, Univ. of Pittsburgh, Pittsburgh, PA, USA.

2. The ExOne Company, North Huntingdon, PA, USA.

3. Quintus Technologies, OH, USA.

* Corresponding authors: chmielus@pitt.edu, kak272@pitt.edu

Additive manufacturing (AM) is increasingly adapted to produce custom metal parts. Binder jet printing (BJP) is of great interest due to the ability to make complex parts without the use of a high-power laser or electron beam. In BJP, thin powder layers are placed and spread with a roller. Binder is deposited onto each layer to temporarily bind shapes (Figure 1a). After printing, samples are sintered and potentially treated with hot isostatic pressing (HIP) to densify the printed part. HIP uses an inert gas to apply a high pressure at an elevated temperature which allows the internal pores to overcome the surface energy of the powders and reduce the porosity (Figure 1b) [1].

316L stainless steel (316L) is heavily used in both structural and biomedical applications [2,3], making it important to understand its fatigue behavior. In powder metallurgy, HIP is beneficial in controlling the final resulting porosity which makes it ideal for AM as well $[1,2,4]$. As AM and BJP is increasingly adapted, a better understanding of the influence of sintering and HIP on mechanical properties, especially fatigue, of BJP 316L parts is crucial since little research currently available [5,6]. Here, we investigate the effects of HIP cycles on fatigue behavior of BJP manufactured 316L and compare it to the as-sintered and literature values of traditionally manufactured 316L.

100 fatigue samples were printed from $-38+5 \mu \mathrm{m}$ Sandvik $316 \mathrm{~L}$ powder using an ExOne M-Flex binder jet printer. All 100 samples were sintered with ExOne's recommended profile $\left(1380^{\circ} \mathrm{C}\right)$, and 80 samples received one of the additional four different HIP treatments (20 each, Table 1). Fatigue behavior was tested with an MTS Landmark load frame at 9 stress levels from 150-350 MPa in $25 \mathrm{MPa}$ increments at $15 \mathrm{~Hz}$ and $\mathrm{R}=-1$. Fractography was performed with a Zeiss SmartZoom5 optical microscope.

Fatigue results shown in Fig. 2a include fatigue results from a $\mathrm{R}=0.1$ test on metal injection molding (MIM) parts [2] and a $\mathrm{R}=0.1$ test on machined 316L [5]. Both the HIP and sintered parts showed a better overall fatigue life $(\mathrm{R}=-1)$ than the less-aggressive tension-tension test of MIM parts $(\mathrm{R}=0.1)$. Interestingly, there was no significant fatigue life difference between the sintered and the various HIP parts. However, there was a difference in the spread of the cycles-to-failure per stress level. For the sintered parts, the $\mathrm{R}^{2}$ was 0.88 , for all HIP parts $\mathrm{R}^{2}>0.93$, even as high as 0.95 for HIP 1,2 . The remaining porosity is likely more uniform for HIP, resulting in less spread of cycle-to-failure numbers.

Fractography on samples of high, medium, and low stress levels (Figure 2b) show crack initiation sites (arrows), and lines separate the region II (stable crack growth) and III (rupture). Dark lines in region II are a result of two cracks that join to form a step-like feature (ratchet mark) [7]. Fewer ratchet marks are seen in the low-stress vs. high-stress samples. Additionally, region II increases with increasing load. The high number of initiation points is likely due to the high surface roughness. Crack initiation on the surface suggests that internal structure, a result of HIP treatment, does not have an effect on part strength. In order 
to determine the true effect of HIP on fatigue strength, surface defects would have to be removed.

In conclusion, BJP parts have fatigue properties better than MIM and similar to machined parts. HIP does not affect the average fatigue strength, but it does improve the cycle-to-failure consistency of sintered BJP parts. Abundant cracking due to high surface roughness can be reduced in future work by mechanically polishing the surface to decrease roughness and improve fatigue strength $[6,8]$

References:

[1] A Kumar et al., Proc. Manuf. 10 (2017), p. 935.

[2] K Essa et al., Mat. \& Des. 138 (2018), p. 21.

[3] Y Zhang et al., Metals. 8 (2018), p. 893.

[4] A Kumar et al., Additive Manuf. 24 (2018), p. 115.

[5] KA Mohammad et al., IOP Conf. Series: Mat. Sci. and Eng. 36 (2012), p. 012012.

[6] A Mostafaei et al., Additive Manuf. 24 (2018), p. 200.

[7] WT Becker, Failure Analysis and Prevention. (2002), p. 559.

[8] The authors would like to thank Xiaoyi Liu, Pierangeli de Vecchis Rodriguez for fatigue testing and the Materials Micro-Characterization Laboratory at the University of Pittsburgh for characterization.

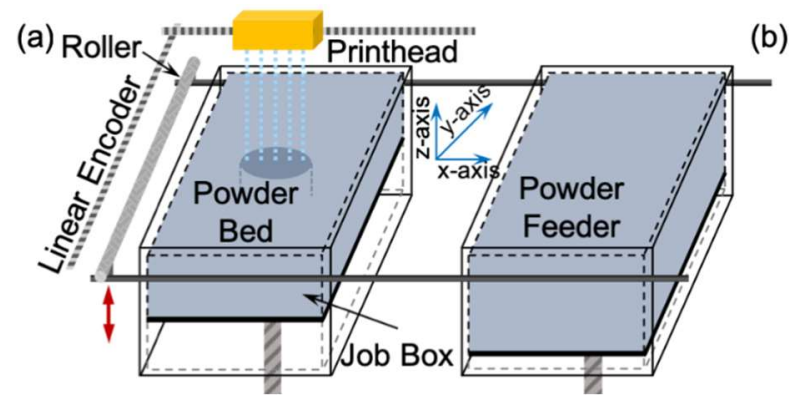

(b)

Figure 1. Schematics showing production methods of (a) BJP and (b) HIP.
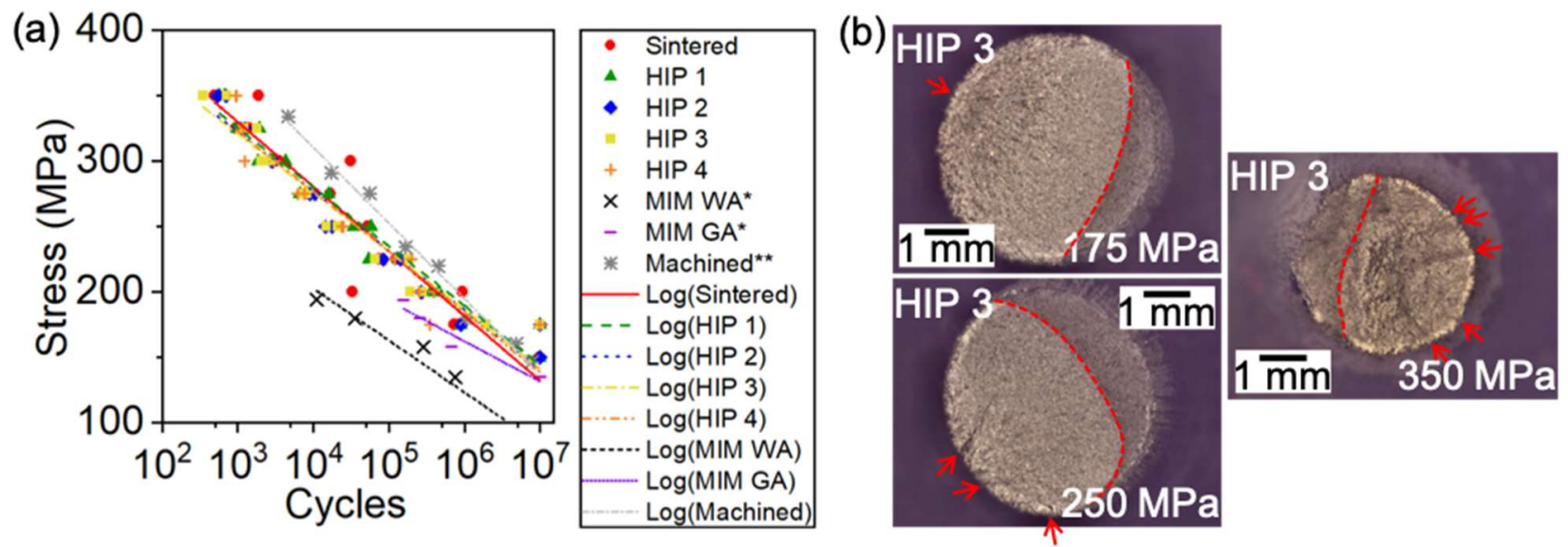

Figure 2. Results of (a) fatigue testing and (b) optical micrographs of representative fracture surfaces.

\begin{tabular}{cccc}
\hline Condition & Temp $\left({ }^{\circ} \mathrm{C}\right)$ & Pressure $(\mathrm{MPa})$ & Time $(\mathrm{min})$ \\
\hline Sintered & 1380 & N/A & 240 \\
HIP 1 & 1130 & 150 & 75 \\
HIP 2 & 1165 & 150 & 75 \\
HIP 3 & 1130 & 150 & 75 \\
HIP 4 & 1165 & 150 & 75 \\
\hline
\end{tabular}

Table 1. Sintering and HIP conditions. 